\title{
Long-term outcomes of patients who underwent limited proximal gastrectomy
}

\author{
Daisuke Ichikawa $\cdot$ Shuhei Komatsu $\cdot$ Takeshi Kubota $\cdot$ \\ Kazuma Okamoto $\cdot$ Atsushi Shiozaki $\cdot$ Hitoshi Fujiwara $\cdot$ \\ Eigo Otsuji
}

Received: 17 December 2012/Accepted: 15 March 2013/Published online: 5 April 2013

(c) The International Gastric Cancer Association and The Japanese Gastric Cancer Association 2013

\begin{abstract}
Background Because the incidence of early gastric cancers has been increasing in Asian countries, postoperative quality of life has received considerable attention in addition to oncological outcomes.

Methods Eighty-four patients with clinically early gastric cancers were enrolled in this retrospective study. Among them, 35 patients underwent total gastrectomy (TG) and 49 patients underwent limited proximal gastrectomy (PG). Blood chemistry, changes in body weight, and oncological outcomes were compared between the two groups.

Results Postoperative hemoglobin levels and body weights were significantly lower in the TG group than in the PG group, and there were no significant differences in the levels of other nutritional indicators such as serum total protein and total cholesterol. However, the overall survival rates of patients in the PG group were similar to those of patients in the TG group (5-year survival rates, 95 versus $97 \%$, respectively; $p=0.86$ ).

Conclusions Limited proximal gastrectomy with regional lymph node dissection has possible positive effects on maintaining body weight and preventing postgastrectomy anemia with similar oncological outcomes to total gastrectomy in patients with early gastric cancers.
\end{abstract}

D. Ichikawa $(\bowtie) \cdot$ S. Komatsu - T. Kubota · K. Okamoto ·

A. Shiozaki · H. Fujiwara · E. Otsuji

Division of Digestive Surgery, Department of Surgery,

Kyoto Prefectural University of Medicine, 465 Kajii-cho,

Kamigyo-ku, Kyoto 602-8566, Japan

e-mail: ichikawa@koto.kpu-m.ac.jp
Keywords Proximal gastrectomy · Outcomes · Anemia · Body weight

\section{Introduction}

The number of early gastric cancers in the upper third of the stomach has been increasing recently in Asian countries $[1,2]$. Previously, extensive resection such as total gastrectomy with extensive lymph node dissection was performed even for relatively early disease. However, several recent reports have demonstrated that limited proximal gastrectomy with regional lymph node dissection could achieve oncological outcomes equivalent to those of total gastrectomy, at least in patients with upper-third gastric cancer [3, 4].

Postoperative quality of life has received considerable attention in addition to oncological outcomes. However, the advantages of proximal gastrectomy remain a matter of debate $[5,6]$. Because reflux esophagitis is one of the most troublesome complications following proximal gastrectomy, jejunal interposition reconstruction has been the preferred option at many hospitals [7-9]. On the other hand, recent studies advocated that direct esophagogastrostomy with some modifications facilitated an uncomplicated postoperative course similar to or better than jejunal interposition [10-12].

We previously reported favorable postoperative shortterm results for direct end-to-side esophagogastrostomy with bilateral anchoring seromuscular sutures creating an acute angle at the anastomosis [12-14]. In this study, we attempted to clarify the long-term benefits of limited proximal gastrectomy in patients with clinically early gastric cancers in the upper third of the stomach. 


\section{Materials and methods}

\section{Patients}

Between 1999 and 2011, a total of 1,442 patients with gastric cancer were admitted to Kyoto Prefectural University of Medicine. During this period, 110 patients with clinically early gastric cancer (cT1) in the upper third of the stomach underwent proximal gastrectomy (PG) followed by esophagogastrostomy reconstruction or total gastrectomy (TG) followed by Roux-en-Y reconstruction. In principle, the indication for $\mathrm{PG}$ was gastric cancer located in the upper third of the stomach with a preoperative clinical stage of $\mathrm{T} 1 \mathrm{~N} 0$, for which a resection line was planned at least $5 \mathrm{~cm}$ apart from the gastric angle. However, the final decisions on surgical procedures were left to the primary surgeons. After excluding patients lost during follow-up and those who died of other diseases, 84 patients were enrolled in this retrospective study [median follow-up period, 56 months (range 5-159 months), in November 2012). Of these patients, 35 patients underwent TG and 49 patients underwent PG, with each median follow-up period being 41 months (range 5-159 months) and 78 months (range 6-156 months), respectively.

Blood chemistry and changes in body weight were compared between the two groups, and survival rates were also analyzed based on follow-up data as of November 2012. Preoperative clinical diagnoses of tumor invasions were generally given based on the findings of endoscopy, ultrasound endoscopy, and barium study. The clinicopathological features of these patients were reviewed retrospectively from hospital records. Macroscopic and microscopic classifications of gastric cancers were based on the Japanese Classification of Gastric Carcinoma [15].

Surgical procedures

The details of our reconstruction after proximal gastrectomy have been previously described [12-14]. In brief, esophagogastrostomy anastomosis was performed using a circular stapler in an end-to-side manner at the site of the anterior wall after limited proximal gastrectomy (one third of the stomach) with regional lymph node dissection. This technique allowed the greater curvature near the top of the remnant stomach to function as the new fundus. Finally, a few seromuscular anchoring sutures were made between the top of the remnant stomach and the lower esophagus on both sides. Total gastrectomy was broadly performed as previously described [12].

\section{Statistical analysis}

Chi-square tests for categorical variables and the Student $t$ test for unpaired data for continuous variables were performed to compare clinicopathological characteristics between the two groups. A $p$ value of $<0.05$ was regarded as significant. Survival rates were calculated by the Kaplan-Meier method, with the date of gastrectomy as the starting point. Differences in survival were examined by the log-rank test.

\section{Results}

The mean patient age was 64 years (range 36-87), and the male:female ratio was 2.9:1. Median tumor size was $30 \mathrm{~mm}$ (range 5-100 mm). The clinicopathological characteristics of the two groups are shown in Table 1. Patients in the TG group had significantly larger tumors $(p \leq 0.01)$ that tended to be deeper tumors ( $p=0.096$ ) than those of patients in the PG group. However, there were no significant differences in age, gender, tumor macroscopic type, histology, or lymph node metastasis between the two groups. On the other hand, there was no significant difference in postoperative complications between the TG and PG groups (Table 1).

Table 1 Clinicopathological characteristics

\begin{tabular}{|c|c|c|c|}
\hline & $\begin{array}{l}\text { Proximal } \\
\text { gastrectomy (PG) }\end{array}$ & $\begin{array}{l}\text { Total } \\
\text { gastrectomy } \\
\text { (TG) }\end{array}$ & $p$ value \\
\hline \multicolumn{4}{|l|}{ Age (years) } \\
\hline Mean (range) & $63(36-80)$ & $65(42-87)$ & 0.31 \\
\hline \multicolumn{4}{|l|}{ Sex } \\
\hline Male & 34 & 29 & \\
\hline Female & 15 & 6 & 0.16 \\
\hline \multicolumn{4}{|c|}{ Macroscopic findings } \\
\hline Localized & 12 & 6 & \\
\hline Diffuse & 37 & 29 & 0.42 \\
\hline \multicolumn{4}{|l|}{ Tumor size } \\
\hline Mean (range) & $24(5-65)$ & $38(8-100)$ & $<0.01$ \\
\hline \multicolumn{4}{|l|}{ Histology } \\
\hline Differentiated & 32 & 20 & \\
\hline Undifferentiated & 17 & 15 & 0.45 \\
\hline \multicolumn{4}{|l|}{$\mathrm{pT}$} \\
\hline pT1a & 14 & 8 & \\
\hline pT1b & 30 & 17 & \\
\hline $\mathrm{pT} 2<$ & 5 & 10 & 0.096 \\
\hline \multicolumn{4}{|l|}{$\mathrm{pN}$} \\
\hline Negative & 46 & 28 & \\
\hline Positive & 3 & 7 & 0.053 \\
\hline \multicolumn{4}{|c|}{ Postoperative complications } \\
\hline Leakage & 1 & 1 & \\
\hline $\begin{array}{l}\text { Pancreatic } \\
\text { fistula }\end{array}$ & 2 & 2 & \\
\hline Stenosis & 3 & 1 & 0.89 \\
\hline
\end{tabular}


Fig. 1 Comparison of nutritional outcomes between proximal gastrectomy (PG) and total gastrectomy (TG). All postoperative data are represented as values (mean $\pm \mathrm{SE}$ ) relative to preoperative data. There was no significant difference in postoperative levels of serum total protein (a) and total cholesterol (b). Serum hemoglobin levels (c) and body weights (d) were significantly lower in the TG group than in the PG group. ${ }^{*} p<0.05$, $* * p<0.01$, significant

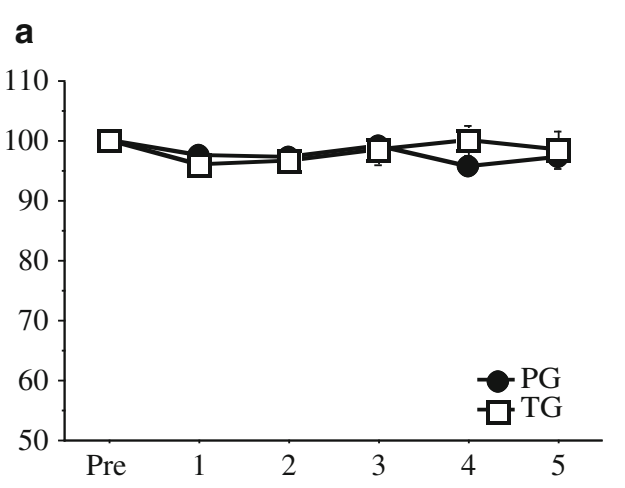

\section{b}
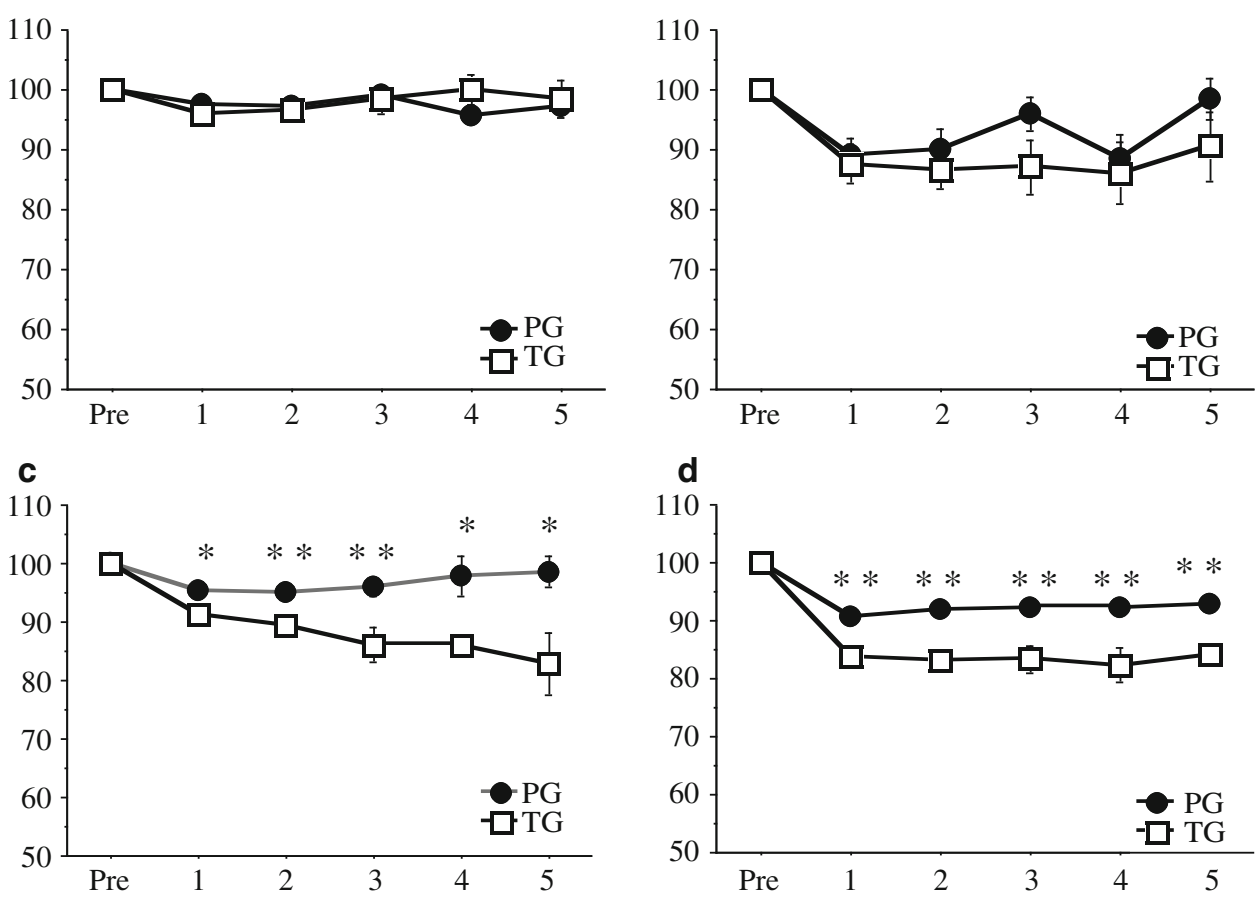

Fig. 2 Overall survival of patients in both groups. There were no significant differences in the overall survival rates of patients in PG and TG groups (5-year survival rates, 95 versus $97 \%$, respectively; $p=0.86$ )

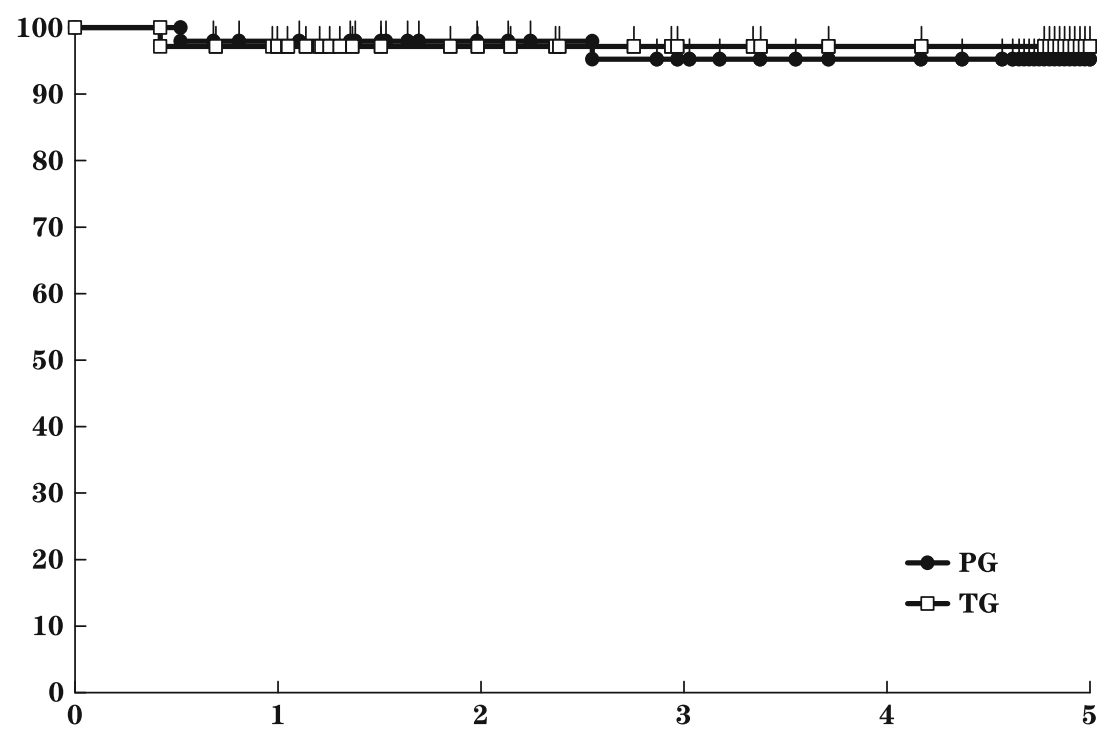

Blood chemistry findings reflecting nutritional status, such as total protein and total cholesterol levels, were not significantly different between the two groups. However, hemoglobin levels were significantly lower in the TG group than in the PG group. This difference tended to expand gradually, although two patients in the TG group received vitamin $B_{12}$ replacement therapy before 5 years post surgery. Moreover, body weights were significantly lower in the TG group than in the PG group (Fig. 1).

Regarding prognoses, one patient died of postoperative complications, and recurrence developed in only one patient in the PG group, who had 15 metastatic lymph nodes at the primary operation and developed peritoneal and bone metastases. The overall survival rate of the PG group was similar to that of TG group (5-year survival rates, 95 versus $97 \%$, respectively; $p=0.86$ ) (Fig. 2).

\section{Discussion}

Because the incidence of early gastric cancers has been increasing worldwide $[1,2,16]$, postoperative quality of 
life has received considerable attention in addition to oncological outcomes. The detection of early gastric cancers in the upper third of the stomach in particular has been increasing in Asian countries [1,2]; therefore, proximal gastrectomy has been recommended as a function-preserving limited operation. However, there is still controversy as to whether proximal gastrectomy is superior to total gastrectomy in terms of postoperative functions, even though they have been considered to achieve similar prognostic outcomes [3-6].

Our concept of proximal gastrectomy consists of the preservation of at least two thirds of the stomach, prevention of reflux esophagitis by end-to-side anastomosis, using an anchoring suture to create an acute angle at the anastomosis, and creation of a substitutional fundus. Although direct esophagogastrostomy had been considered to frequently increase reflux esophagitis, several recent reports including ours have demonstrated that optimal additional procedures could prevent objective endoscopic findings and also postoperative patients' symptoms of reflux disease [10-14]. Recently, limited operations, such as proximal gastrectomy, are more likely to be performed in a laparoscopic approach, and laparoscopic proximal gastrectomy could result in similar short-term outcomes to those of the conventional open method $[10,11,14]$.

The present study investigated the long-term outcomes of our proximal gastrectomy for early gastric cancers in the upper third of the stomach. For oncological outcomes, the survival rate of the PG group was similar to that of the TG group, which is consistent with previous reports [3, 4]. In the PG group of this study, one patient developed peritoneal and hematogenous recurrence. However, this case was considered to have tumors of extremely progressive characteristics with widespread lymph node metastases at the time of surgery; therefore, the eradication of such exceptional tumors with multiple distant lymph node metastases seemed almost impossible even with total gastrectomy and extended lymphadenectomy. These observations suggest that proximal gastrectomy with regional lymphadenectomy is usually satisfactory in early gastric cancers in the upper third of the stomach.

Postoperative nutritional status has also been one of the main issues for patients who have undergone gastrectomy. Nutrition deficiencies are common after total gastrectomy. The present results showed the possible positive effects of proximal gastrectomy on maintaining body weight and preventing postgastrectomy anemia relative to total gastrectomy, although there were no improvements in the indicators of nutritional status, such as serum total protein and total cholesterol. Loss of body weight after gastrectomy is related to reductions in oral food intake and impaired absorption. As shown in our previous report, extreme reductions in the volume of food intake were more frequently experienced in patients who underwent total gastrectomy than in those who underwent proximal gastrectomy [13]. Moreover, our other recent report using a questionnaire survey showed that the indigestion syndrome was more frequently seen in the TG group than in the PG group, which indicates that absorption is more impaired in the TG group [12]. The effect of proximal gastrectomy on the maintenance of body weight may be explained in terms of the aforementioned findings. An et al. [6] reported that proximal gastrectomy provided no benefit in terms of postoperative weight loss. However, they reported that their PG series were associated with a markedly higher rate of anastomotic stenosis and reflux esophagitis, which may have reduced oral food intake. The complication rate of our previously reported study was not as high as theirs; therefore, this discrepancy in results between the present study and theirs may be caused by differences in complication rates.

For postoperative anemia, patients in the PG group have theoretically more obvious advantages for the possible secretion of gastric intrinsic factor from residual gastric mucosa cells than those in the TG group. Because the hepatic and pyloric branches of the vagus nerve are preserved in our proximal gastrectomy procedure, we believe that the function of gastric acid secretion could also be preserved to a degree. As expected, the present results clearly demonstrated that PG is superior in terms of preventing postoperative anemia, and differences in hemoglobin levels between the two groups became pronounced over time. However, An et al. [6] demonstrated that hemoglobin levels were similar between patients who underwent gastric tube reconstruction after proximal gastrectomy and those who underwent a Roux-en Y reconstruction after total gastrectomy. Although the reason for the discrepancy between our results and theirs is not known exactly, it may be associated with the volume and/or acid secretion function of remnant gastric cells.

In conclusion, limited proximal gastrectomy with regional lymph node dissection could achieve similar oncological outcomes to total gastrectomy, at least in patients with early gastric cancers, and may also have possible positive effects on maintaining body weight and preventing postgastrectomy anemia.

\section{References}

1. Okabayashi T, Gotoda T, Kondo H, et al. Early carcinoma of the gastric cardia in Japan: is it different from that in the West? Cancer (Phila). 2000;89:2555-9.

2. Ahn HS, Lee HJ, Yoo MW, et al. Changes in clinicopathological features and survival after gastrectomy for gastric cancer over a 20-year period. Br J Surg. 2011;98:255-60. 
3. Harrison LE, Karpeh MS, Brennan MF. Total gastrectomy is not necessary for proximal gastric cancer. Surgery (St. Louis). 1998;123:127-30.

4. Katai H, Morita S, Saka M, et al. Long-term outcome after proximal gastrectomy with jejunal interposition for suspected early cancer in the upper third of the stomach. Br J Surg. 2010;97:558-62.

5. Shiraishi N, Adachi Y, Kitano S, et al. Clinical outcome of proximal versus total gastrectomy for proximal gastric cancer. World J Surg. 2002;26:1150-4.

6. An JY, Youn HG, Choi MG, et al. The difficult choice between total and proximal gastrectomy in proximal early gastric cancer. Am J Surg. 2008;196:587-91.

7. Takeshita K, Saito N, Saeki I, et al. Proximal gastrectomy and jejunal pouch interposition for the treatment of early cancer in the upper third of the stomach: surgical technique and evaluation of postoperative function. Surgery (St. Louis). 1997;121:278-86.

8. Namikawa T, Oki T, Kitagawa H, et al. Impact of jejunal pouch interposition reconstruction after proximal gastrectomy for early gastric cancer on quality of life: short- and long-term consequences. Am J Surg. 2012;204:203-9.

9. Tokunaga M, Ohyama S, Hiki N, et al. Endoscopic evaluation of reflux esophagitis after proximal gastrectomy: comparison between esophagogastric anastomosis and jejunal interposition. World J Surg. 2008;32:1473-7.
10. Hiki N, Fukunaga T, Yamaguchi T, et al. Laparoscopic esophagogastric circular stapled anastomosis: a modified technique to protect the esophagus. Gastric Cancer. 2007;10:181-6.

11. Sakuramoto S, Yamashita K, Kikuchi S, et al. Clinical experience of laparoscopy-assisted proximal gastrectomy with Toupet-like partial fundoplication in early gastric cancer for preventing reflux esophagitis. J Am Coll Surg. 2009;209:344-51.

12. Ichikawa D, Komatsu S, Okamoto K, et al. Evaluation of symptoms related to reflux esophagitis in patients with esophagogastrostomy after proximal gastrectomy. Langenbecks Arch Surg. 2013. doi:10.1007/s00423-012-0921-0.

13. Ichikawa D, Ueshima Y, Shirono K, et al. Esophagogastrostomy reconstruction after limited proximal gastrectomy. Hepatogastroenterology. 2001;48:1797-801.

14. Ichikawa D, Komatsu S, Okamoto K, et al. Esophagogastrostomy using a circular stapler in laparoscopy-assisted proximal gastrectomy with an incision in the left abdomen. Langenbecks Arch Surg. 2012;397:57-62.

15. Japanese Gastric Cancer Association. Japanese classification of gastric carcinoma: 3rd English edition. Gastric Cancer. 2011;14:101-12.

16. Triantafillidis JK, Cheracakis P. Diagnostic evaluation of patients with early gastric cancer-a literature review. Hepatogastroenterology. 2004;51:618-24. 